\title{
A novel orange-colored bimolecular fluorescence complementation (BiFC) assay using monomeric Kusabira-Orange protein
}

\author{
Yuta Fujiii, ${ }^{*}$, Ayaka Yoshimura*,1, and Yutaka Kodama ${ }^{1}$ \\ ${ }^{1}$ Center for Bioscience Research and Education, Utsunomiya University, Tochigi, Japan \\ *Yuta Fujii and Ayaka Yoshimura contributed equally to this work \\ BioTechniques 64:153-161 (April 2018) doi 10.4155/btn-2017-0121 \\ Keywords: BiFC competition assay; Gateway cloning; KO protein; multicolor BiFC; pGWT35S; protein interaction
}

The bimolecular fluorescence complementation (BiFC) assay was developed as a tool for the visualization of protein-protein interactions in living cells. To date, many types of BiFC systems with distinct colors have been developed. Most of the colors in the visible spectrum have been used in BiFC assays, with the exception of orange. In this study, we developed an orange-colored BiFC system using the Kusabira-Orange (KO) protein from the stony coral Fungia concinna. To obtain bright BiFC fluorescence, we compared fluorescence intensities of two monomeric $\mathrm{KO}$ variants ( $\mathrm{mKO} 1$ and $\mathrm{mKO} 2)$ and identified $\mathrm{mKO} 2$ as brighter than mKO1. The optimal split site for mKO2-based BiFC was defined by a comparative analysis of complementation efficiency and a signal-to-noise ratio. The resulting mKO2-based BiFC system successfully demonstrated protein dimerization in plant cells as a model experiment. The novel mKO2-based BiFC system will expand the possibility of multicolor BiFC analysis.

Protein-protein interactions are fundamental to cellular functions. The bimolecular fluorescence complementation (BiFC) assay was developed to visualize protein interactions in living cells [1]. In the BiFC assay, a fluorescence signal can be monitored when two non-fluorescent fragments of a fluorescent protein are brought into proximity by the interacting proteins fused to the fragments. This assay has a wide variety of applications in multicolored systems for simultaneously visualizing multiple protein complexes $[2,3]$. To date, many types of fluorescent proteins with distinct colors (e.g. blue, cyan, green, yellow, red, and far-red), which are derived from the jellyfish Aequorea victoria and coral species, have been utilized in the BiFC system $[3,4]$. However, no system with an orange-colored region (range from $540 \mathrm{~nm}$ to $570 \mathrm{~nm}$ ) has been reported (Supplementary Figure S1).
Kusabira-Orange $(\mathrm{KO})$ is an orange fluorescent protein isolated from the stony coral Fungia concinna, which is known as Kusabira-ishi in Japanese [5]. Although the original clone from $F$. concinna lacks the essential amino acids for fluorescence emission, the mutated $\mathrm{KO}$ (KO1) emits orange fluorescence (excitation peak at 548 nm, emission peak at 561 nm; Supplementary Figure S1) and has remarkable $\mathrm{pH}$ stability (5). Because KO1 forms dimers, monomeric KO1 (mKO1) (excitation peak at $548 \mathrm{~nm}$, emission peak at $559 \mathrm{~nm}$ ) was developed by amino acid substitutions [5]. Subsequently, the fast-folding version of mKO1, named mKO2 (excitation peak at $551 \mathrm{~nm}$, emission peak at $565 \mathrm{~nm}$ ), was generated by introducing eight mutations [6]. The additional mutations in $\mathrm{mKO} 2$ provided faster maturation with comparable spectral characteristics, although it had lower pH stability $[6,7]$. Based on the excitation and emission spectra, $\mathrm{KO}$ proteins are ideal candidates for an orangecolored BiFC system.

To establish a KO-based BiFC system, we evaluated the fluorescence intensity of full-length $\mathrm{KO}$ variants ( $\mathrm{mKO} 1$ and $\mathrm{mKO} 2$ ) using transient expression in mesophyl protoplasts of Arabidopsis thaliana. Furthermore, to define the optimal split site for $\mathrm{KO}$-based BiFC, the complementation efficiency and signal-to-noise $(\mathrm{S} / \mathrm{N})$ ratio were evaluated. The present study demonstrates the novel orange-colored BiFC system and provides valuable information for in planta imaging with $\mathrm{KO}$ variants.

\section{Materials and methods}

Plasmid construction

To construct expression vectors for fulllength $\mathrm{KO}$ variants (KO1, mKO1, and $\mathrm{mKO}$ ) and humanized $\mathrm{KO}$ variants (hKO1, hmKO1, and hmKO2) (Medical and Biological Labora-

\section{METHOD SUMMARY}

To establish an orange-colored BiFC assay, we employed the fragments from monomeric Kusabira-Orange 2 (mKO2). The mKO2-based BiFC system demonstrated protein dimerization in plant cells (Arabidopsis thaliana). We also performed a BiFC competition assay to estimate the signal-to-noise ratio of the mKO2-based BiFC system. 
tories Co. Ltd, Aichi, Japan), the In-Fusion HD Cloning Kit (Takara Bio Company, Shiga, Japan) was used. The DNA fragments were amplified by PCR with following primers: 5'-GACTCTAGAGGATCCATGGTGAGTGTGATT-3' (F1) and 5'-AGCCGGGCGGCCGCTTTAGCAATGAGCTAC-3' (R1) for KO1, F1 and 5'AGCCGGGCGGCCGCTTTAGGAATGAGCTAC-3' (R2) for mKO1 and mKO2, 5'-GACTCTAGAGGATCCATGGTGAGCGTGATC-3' (F2) and 5'-AGCCGGGCGGCCGCTTTAGCAGTGGGCCAC-3' (R3) for hKO1, and F2 and 5'-AGCCGGGCGGCCGCTTTAGGAGTGGGCCAC-3' (R4) for hmKO1 and hmKO2. The PCR-amplified DNA fragments were replaced with GREEN FLUORESCENTPROTEIN-S65T (GFP-S65T) of the P35S-GFP-S65T-Tnos vector by the In-Fusion reaction [8]. For construction of vectors for mKO2-based BiFC, Gateway Cloning Technology (Thermo Fisher Scientific, Waltham, USA) was used. The DNA fragments encoding $\mathrm{N}$ - or C-terminal fragments of mKO2 (KON154, KON168, KON206, KOC155, KOC169, and KOC207) were introduced into the pDONR207 vector by BP recombination reaction (Thermo Fisher Scientific). The DNA fragments were amplified by PCR using the following primers: 5'-GGGGACAAGTTTGTACAAAAAAGCAGGCTTCATGGTGAGTGTGATTAAA-3' (F3) and 5'-GGGGACCACTTTGTACAAGAAAGCTGGGTCTTATCCGTCGCTGGCAGTA-3' (R5) for KON154, F3 and 5'-GGGGACCACTTTGTACAAGAAAGCT GGGTCTTATCCTTCAAGTTTTAGG-3' (R6) for KON168, F3 and 5'-GGGGACCACTTTGTACAAGAAAGCTGGGTCTTAGTTGCCTTCGGTTTTC-3' (R7) for KON206, 5'-GGGGACAAGTTTGTACAAAAAAGCAGGCTTCATGGTTCTGAAGGGTGAT-3' (F4) and 5'-GGGGACCACTTTGTACAAGAAAGCTGGGTCTTAGGAATGAGCTACTGCA-3' (R8) for KOC155, 5'-GGGGACAAGTTTGTACAAAAAAGC AGGCTTCATGGGCGGCAATCACAAA-3' (F5) and R8 for KOC169, and 5'-GGGGACAAGTTTGTACAAAAAAGCAGGCTTCATGATTACTGAGCAGGTA-3' (F6) and R8 for KOC207. Subsequently, using the LR recombination reaction (Thermo Fisher Scientific), the inserted DNA fragments were transferred into the pGWT35S vector, which is a new synthetic vector for transient expression in plant cells (Supplementary Figure S2) that is optimized for Gateway Cloning Technology (Thermo Fisher Scientific). We designed the pGWT35S vector, and Operon Technologies Inc. constructed it (Alameda,
USA). The pGWT35S vector backbone, excluding the Gateway cassette (ccdB and chloramphenicol resistance genes), is compact (3,533 bp) as is the backbone of the P35S-sfGFP-Tnos vector (3,463 bp) [8], and it is applicable for protein-tagging methods using the GGSGGS linker peptides following the attR sequences (Supplementary Figure S2). To fuse 7-methylxanthine methyltransferase (MXMT) with the BiFC fragments, the DNA fragments for MXMT were amplified by PCR using the following primers: 5'-ATATCCATGGATGGAGCTCCAAGAAGTCCTGCATA-3' (F7) and 5'-ATATACTAGTTTACACGTCTGACTTCTCTGGCTTT-3' (R9). Subsequently, the MXMT gene was subcloned into the $\mathrm{Ncol} /$ Spel site of the resulting vectors encoding the BiFC fragments.

\section{Transient expression assay}

Seeds of Arabidopsis thaliana containing gl-1 mutation were grown in vermiculite under white light (approximately $80 \mu \mathrm{mol}$ photons $\mathrm{m}^{-2} \mathrm{~s}^{-1}$ ) with a light (16 h)/dark (8 h) cycle at $22^{\circ} \mathrm{C}$ in a growth chamber for 3 weeks. Mesophyll protoplasts were prepared and transient expression was performed by the Tape-Arabidopsis sandwich method [9] and polyethylene glycol (PEG)-mediated protoplast transformation, as previously described [8]. Briefly, $100 \mu \mathrm{L}$ of protoplast suspensions (0.4 mM mannitol, 4 mM MES- $\mathrm{NaOH}$ pH 5.7, $15 \mathrm{mM} \mathrm{MgCl}_{2}$ ) were mixed with $5 \mu \mathrm{L}$ of each plasmid DNA $\left(1 \mu^{-1} \mu L^{-1}\right)$ encoding $\mathrm{KO}$ variants and GFP-S65T [8] for the experiments of full-length $\mathrm{KO}$ variants, and were mixed with $4 \mu \mathrm{L}$ of two appropriate BiFC plasmids $\left(1 \mu^{-1} \mu \mathrm{L}^{-1}\right)$ and 2 $\mu \mathrm{L}$ of GFP-S65T plasmid $\left(1 \mu^{-1} \mu \mathrm{L}^{-1}\right)$ for BiFC experiments. Equal volumes of $\mathrm{PEG}$ solutions containing 40\% (v/v) PEG4000 (Fluka, \#81240) with 0.4 M mannitol and $100 \mathrm{mM} \mathrm{CaCl}_{2}$ were added, and the mixture was incubated for $30 \mathrm{~min}$ at $23^{\circ} \mathrm{C}$. After incubation, protoplasts were washed with $5 \mathrm{~mL}$ of W5 solution $(5 \mathrm{mM} \mathrm{KCl}, 2 \mathrm{mM}$ MES $\mathrm{pH}$ 5.7, $125 \mathrm{mM} \mathrm{CaCl}_{2}, 154 \mathrm{mM} \mathrm{NaCl}, 0.9 \%$ glucose) and resuspended with $1 \mathrm{~mL}$ of W5 solution. The mixtures were incubated in a six-well culture plate coated with 1\% BSA at $23^{\circ} \mathrm{C}$ for $24 \mathrm{~h}$ under dark conditions.

\section{BiFC competition assay}

The BiFC competition assay was performed according to a previous report with some modifications [8]. In addition to $4 \mu \mathrm{L}$ each of the two BiFC plasmids $\left(1 \mu \mathrm{g}^{-1} \mu \mathrm{L}^{-1}\right)$ for
mKO2-based BiFC and $2 \mu \mathrm{L}$ of GFP-S65T plasmid for normalization of protein expression, $40 \mu \mathrm{L}$ of plasmid $\left(1 \mu^{-1} \mu \mathrm{L}^{-1}\right)$ coding the fusion protein of MXMT with the C-terminal fragment (155-239 amino acids) of GFP-S65T (GC155-MXMT) $(8,10)$ was added to $100 \mu \mathrm{L}$ of the protoplast solution as a competitor for the BiFC reaction. Subsequently, an equal volume (150 $\mu \mathrm{L}$ ) of PEG solution was added and treated as mentioned above. Instead of the GC155-MXMT plasmid, $40 \mu \mathrm{L}$ of the GC plasmid $\left(1 \mu^{-1} \mu \mathrm{L}^{-1}\right)[8,10]$ was used as a control plasmid for setting to the same transfection efficiency.

\section{Fluorescence microscopy}

Measurements and evaluation of fluorescence intensity were performed as previously reported [8]. Fluorescence images of protoplasts were captured using fluorescence microscopes (Axio Imager Zim, Carl Zeiss, Göttingen, Germany, and BX60, Olympus, Tokyo, Japan) with specific filter sets for KO (excitation, BP 525/45 nm; emission, BP 595/60 nm; dichroic filter, 560DRLP) and GFP (excitation, BP 480/40 nm; emission, BP 535/50 nm). Quantification of fluorescence intensity was performed with ImageJ software [11] as previously described [12]. Briefly, the orange (KO) and green (GFP) fluorescent images of randomly selected 10 protoplasts were stacked by using the ImageJ command "Stack to Images." The background signal of the images was subtracted by using the ImageJ command "Subtract Background," and then intensities of orange $(\mathrm{KO})$ and green (GFP) fluorescence at the cytoplasmic region were measured. The raw data (i.e., actual fluorescence values) measured were shown in Supplemental Tables S1-7. To normalize the protein expression levels, the fluorescence intensity of KO was divided by that of GFP, and a median was obtained. This procedure was repeated three times. Subsequently, both means and standard deviations were calculated from the three medians. For statistical analysis, TukeyKramer test and Student's t-test were performed using R statistical software [13].

\section{Results and discussion}

Full-length $\mathrm{KO}$ variants expressed in Arabidopsis

It is reported that full-length fluorescent proteins, which yield a bright signal under physiological conditions, were preferred 
for BiFC experiments [3]. To evaluate the fluorescence intensity of full-length $\mathrm{KO}$ variants in living cells, the plasmids encoding monomeric $\mathrm{KO}$ protein (mKO1 or $\mathrm{mKO}$ ) were transfected into mesophyll protoplasts of $A$. thaliana. After transfection, fluorescence-emitting protoplasts were randomly selected and analyzed by fluorescence microscopy. The variability of the protein expression level between each cell was normalized by the fluorescence intensity of co-expressed GFP-S65T (Figure 1A), as previously described [8]. When $\mathrm{KO}$ variants were compared, the relative fluorescence intensity of $\mathrm{mKO} 2$ was approximately 12 -fold higher than that of mKO1 (Figure 1B). Thus, $\mathrm{mKO} 2$ was employed to develop the orangecolored BiFC system.

BiFC efficiency of mKO2 fragments with different split sites

Because the structure of $\mathrm{mKO} 2$ is not available, we employed the crystal structure of mKO1 (PDB ID: 3MGF) to design mKO2-based BiFC. In previous reports using GFP variants, the loop structures between the 7 th and 8 th $\beta$-strands or 8th and 9 th $\beta$-strands were commonly used as the split site of BiFC fragments [3] (Figure 2). Additionally, the loop between 10th and 11th $\beta$-strands was successfully used in some BiFC systems using GFP variants $[3,14,15]$. The corresponding split sites were positioned at the loops between 8th and 9th, 9th and 10th, or 11th and 12th $\beta$-strands of mKO1 (Figure 2). Based on the information, we selected three sites (between G154 and V155, G168 and G169, or N206 and 1207) positioned at the loops for the development of mKO2-based BiFC (Figure 2).

To perform the model experiment of mKO2-based BiFC, the MXMT protein from Coffea arabica was employed because MXMT forms a homodimer that can be visualized by the BiFC assay $[2,8,10,16]$. The appropriate pairs of plasmids (KON154-MXMT/KOC155-MXMT, KON168-MXMT/KOC169-MXMT, or KON206-MXMT/KOC207-MXMT) were transfected into protoplasts of $A$. thaliana, and their complemented fluorescence were observed (Figure 3A). Among these pairs, the KON154-MXMT/KOC155-MXMT complex was the brightest (Figure 3, A and B), indicating efficient structural complementation between the KON154 and KOC155 fragments. In contrast, KON168-MXMT/ KOC169-MXMT and KON206-MXMT/
(A)
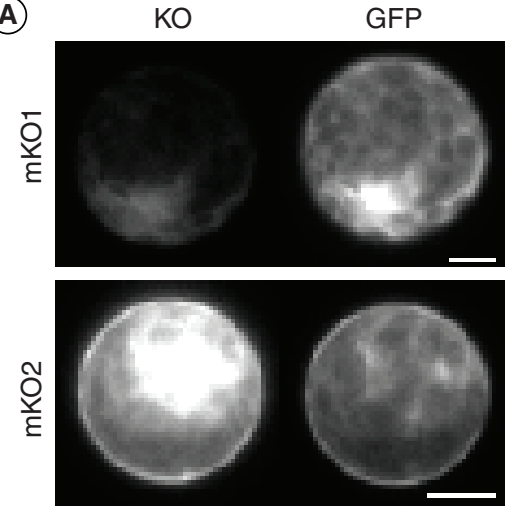

(B)

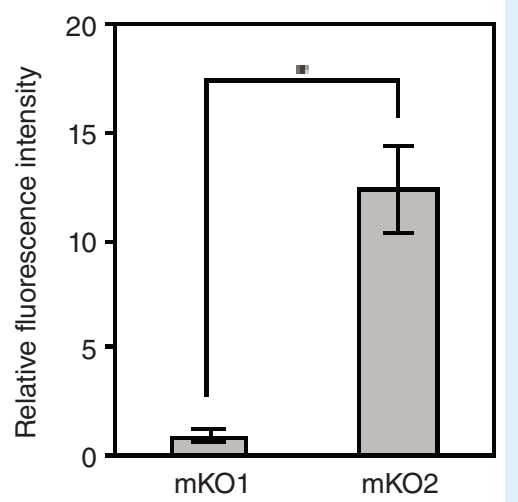

Figure 1. Fluorescence intensity of full-length KO variants. (A) Representative images of a protoplast co-expressing full-length KO variants and GFP-S65T. Bars represent $5 \mu \mathrm{m}$. (B) Relative fluorescence intensity of full-length $\mathrm{KO}$ variants. To normalize the protein expression levels in each cell, fluorescence intensity of KO variants was divided by that of GFP-S65T. The averaged fluorescence intensity of KO variants was normalized to mKO1. Error bars represent standard deviations $(n=3)$. The asterisk indicates statistical significance $(P<0.01)$.

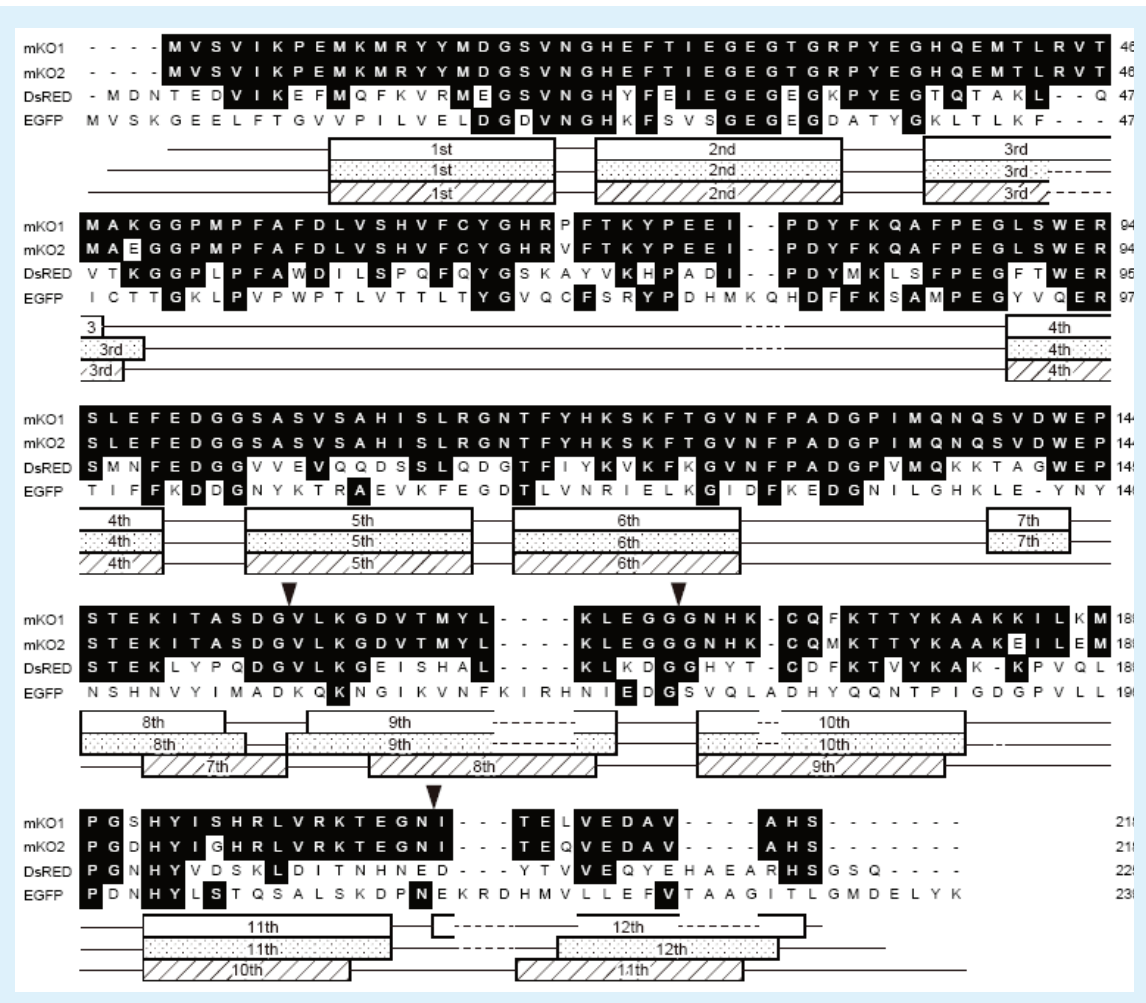

Figure 2. Sequence alignment of mK01, mK02, DsRED monomer, and EGFP. The black box shows identical amino acids. The schematic diagrams below the sequences indicate positions of $\beta$-strands within the secondary structure of mKO1 (PDB ID: 3MGF), DsRED monomer (2VAD) and EGFP (4EUL). The $\beta$-strands are shown as boxes with identical patterns (blank: mKO1, dot: DsRED monomer, stripe: EGFP). The black arrowheads indicate the split sites attempted in the mKO2-based BiFC system.

KOC207-MXMT exhibited dim fluorescence (Figure 3, A and B). Based on these results, splitting mKO2 between $\mathrm{G} 154$ and V155 was determined to be optimal for an mKO2-based BiFC system with high fluorescence intensity.
Estimation of $\mathrm{S} / \mathrm{N}$ ratio of mKO2-based BiFC

Owing to a random collision of non-fluorescent fragments, an undesirable background signal often occurs in BiFC experiments [3]. Although the KON154 and KOC155 pair showed bright fluorescence, 
(A)

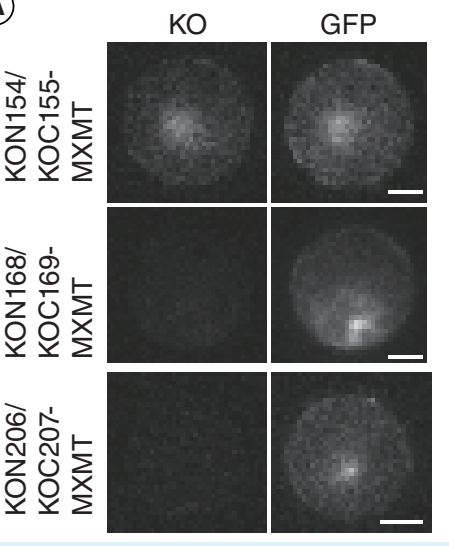

(B)

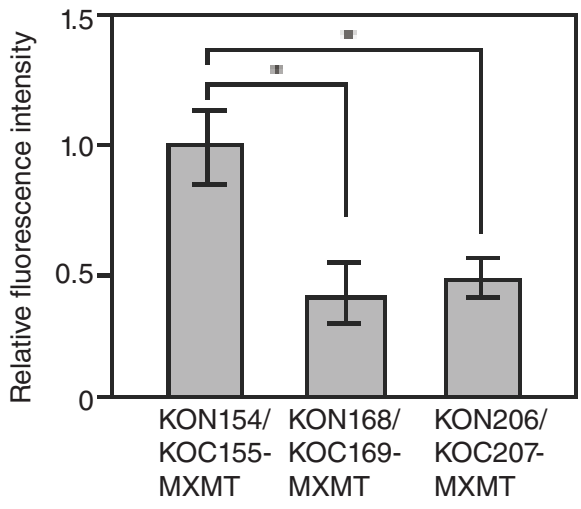

Figure 3. Demonstration of mK02-based BiFC. (A) Representative images of protoplasts co-expressing dimer-formed MXMT-mediated mKO2-based BiFC complex and GFP-S65T. (B) Relative fluorescence intensity of dimer-formed MXMT-mediated mKO2-based BiFC systems. Error bars represent standard deviations $(n=3)$. The asterisks indicate statistical significance $(P<0.01)$.

\section{(A)}

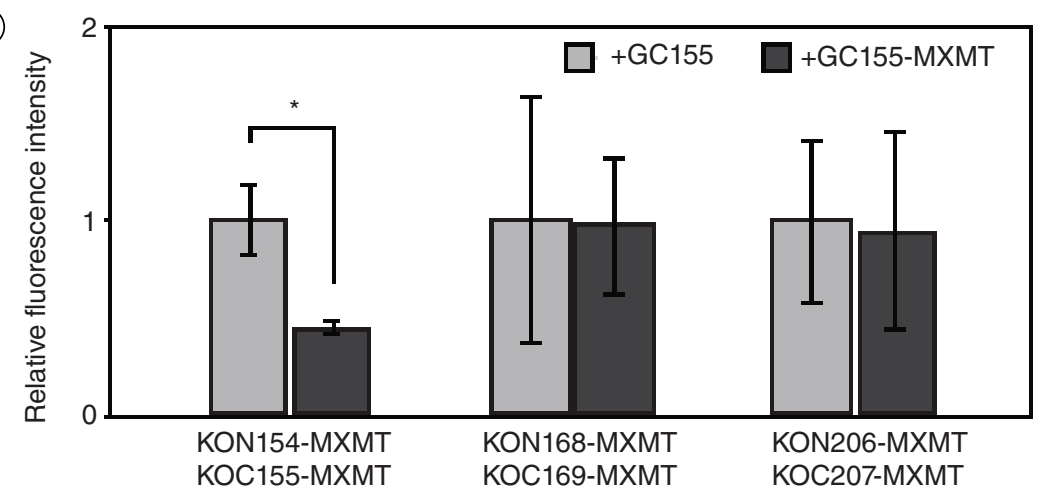

(B)

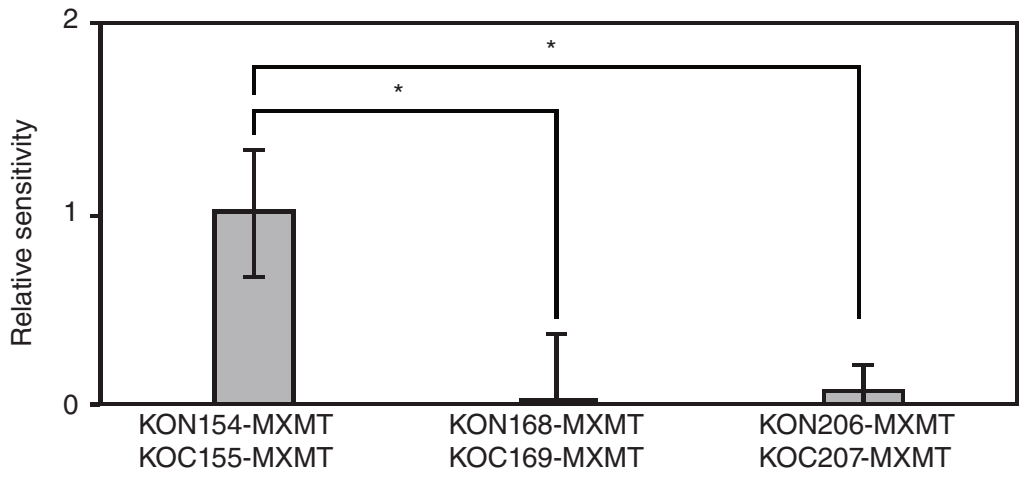

Figure 4. The BiFC competition assay for mK02-based BiFC. (A) In addition to the pairs of BiFC constructs, an MXMT-fused GC155 fragment (GC155-MXMT) was used as a competitor protein (gray bars). Instead of the GC155-MXMT, the GC155 fragment was used for control experiments (black bars). Error bars represent standard deviations $(n=3)$. The asterisk indicates statistical significance $(P<0.01)$ in the Student's t-test. (B) Relative sensitivity of mKO2-based BiFC with different split sites. The degree of competitor-induced fluorescence decrease is shown. Error bars represent standard deviations $(n=3)$. The asterisks indicate statistical significance $(P<0.05)$ in Tukey-Kramer test.

it is possible that frequent self-assembly of fragments results in a higher background signal. To address this, the $\mathrm{S} / \mathrm{N}$ ratio of mKO2-based BiFC was estimated by a BiFC competition assay $[3,8,17,18]$. In this assay, a competitor-induced decrease in BiFC fluorescence reflects the positive signal derived from specific protein-protein interactions and the remaining fluorescence reflects the background signal derived from the self-assembly of BiFC fragments [3]. To estimate the $\mathrm{S} / \mathrm{N}$ ratio of $\mathrm{mKO} 2$-based BiFC, an excess amount of the competitor (GC155-MXMT) and the appropriate plasmid pairs were co-transfected into protoplasts of $A$. thaliana. Instead of GC155-MXMT, the GC155 fragment was used as a negative control. None of the KON fragments exhibited any fluorescence when co-expressed with the GC fragment (Supplementary Figure S3). In the KON168-MXMT/KOC169-MXMT and KON206-MXMT/KOC207-MXMT pairs, the fluorescence intensities were maintained even though the competitor was added (Figure 4A). The competitorinduced decrease in fluorescence intensity was observed only in KON154-MXMT/ KOC155-MXMT, indicating successful detection of dimerization of MXMT (Figure $4 \mathrm{~A})$. The results indicate that $\mathrm{mKO}$-based BiFC with KON154 and KOC155 fragments has the highest $\mathrm{S} / \mathrm{N}$ ratio (i.e., high sensitivity) among these BiFC systems (Figure 4B).

Time-course assessment of BiFC competition assay

Recently, a potential problem of BiFC competition assay was identified [19]. Given the irreversibility of the BiFC reaction, the competitor seems to be out-competed eventually when the BiFC fragments are presented in single cell for a long period [19]. When we expressed the pair of KON154-MXMT/KON155-MXMT (a BiFC complex) and GC-MXMT (a competitor), a significant decrease of BiFC fluorescence was observed after $24 \mathrm{~h}$ (Figure 4 and Figure 5). However, no significant decrease was observed after $42 \mathrm{~h}$ or $84 \mathrm{~h}$ (Figure 5). If out-competition of the competitors occurred at $42 \mathrm{~h}$ or $84 \mathrm{~h}$, a shorter incubation time (i.e., less than $24 \mathrm{~h}$ ) is suitable for performing the BiFC competition assay. However, we could not determine why a significant decrease was not observed at $42 \mathrm{~h}$ or $84 \mathrm{~h}$ because this might also be explained by characteristics of the fusion proteins or conditions of the cells. Further studies with more precisely controlled gene expression systems (e.g., 2in1 vector system) [19] will be needed.

\section{$\mathrm{KO}$ variants in plants}

In the present study, we evaluated the $\mathrm{KO}$ variants in planta, and believe that these variants are valuable for use in plant cells that have bright chloroplast autofluorescence. Autofluorescence often interferes with live imaging of plant cells with exogenous fluorophores due to its high fluorescence 
intensity and broad range spectrum (20). We previously reported an excitation spectrum of chloroplast autofluorescence, and the 520-560 nm region exhibited lower intensity [20]. Because the excitation peak of $\mathrm{mKO} 2$ is at $551 \mathrm{~nm}$, the spectral property seems to keep chloroplast autofluorescence at a low level.

To further develop useful $\mathrm{KO}$ variants for plant cells, the fluorescence intensity should be improved and codon optimization may be effective. Although we did not produce and test $\mathrm{KO}$ protein optimized with a plant codon, humanized-codon variants of $\mathrm{KO}$ (Medical and Biological Laboratories Co., Ltd.) were tested. Surprisingly, the humanized-codon variants exhibited higher fluorescence intensity than the non-humanized codons (Supplementary Figure S4), suggesting that humanized-codon variants are also suitable for practical use in plant cells.

\section{Split sites}

The split site of KON154/KOC155 is positioned at the loop between the 8th and 9th $\beta$-strands, a position that is consistent with those for various of GFP-based BiFC, but not for red fluorescent protein (RFP)based BiFC [3,21]. However, the amino acid sequence surrounding the loop between 8th and 9th $\beta$-strands of $\mathrm{mKO} 2$ is similar to that of RFP variants (Figure 2). Thus, the key factor(s) for determining the optimal split sites for BiFC assays remain to be understood.

\section{Different origins of fluorescent proteins for BiFC systems}

Most of the previous visible-colored BiFC systems rely on fluorescent proteins derived from two species, the jellyfish Aequorea victoria (e.g., EBFP: enhanced blue fluorescent protein, Cerulean, ECFP: enhanced cyan fluorescent protein, EGFP: enhanced GFP, GFP-S65T, folding reporter GFP, superfolder GFP, EYFP: enhanced yellow fluorescent protein, Citrine, and Venus) and the coral Discosoma sp. (e.g., DsRED monomer, mRFP, mCherry, and mKate) [3]. Previous reports suggested that two non-fluorescent fragments with the same origin often cause cross-complementation because of their high sequence similarity [2,3]. In the present study, the $\mathrm{N}$-terminal fragments of $\mathrm{mKO} 2$ did not complement the C-terminal fragments of RFP (RC169) [2] (Supplementary Figure S5), probably due to the distinct amino acid sequences [5] (similarity: 52\%). Moreover, when we tested whether BiFC between

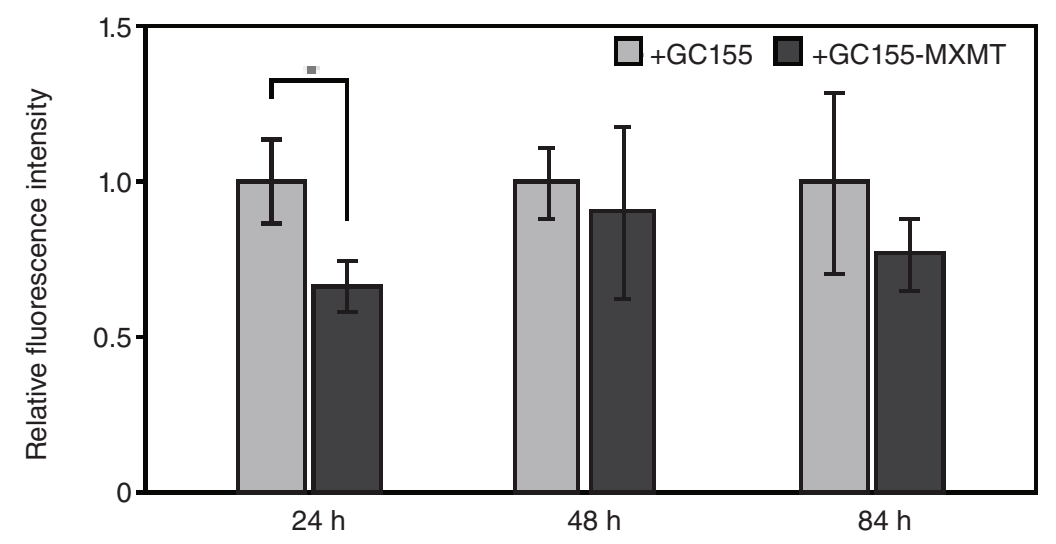

Figure 5. Time-course assessment of BiFC competition assay for mKO2-based BiFC. Relative fluorescence intensities of protoplasts co-expressing pairs of mKO2-based BiFC constructs and MXMT-fused GC155 fragment (GC155-MXMT) as a competitor protein (black bars). Instead of the GC155-MXMT, the GC155 fragment was used for control experiments (gray bars). Fluorescence intensity was measured at 24,48 , or $84 \mathrm{~h}$ after transformation. Error bars represent standard deviations $(n=3)$. The asterisk indicates statistical significance in Student's t-test $(P<0.05)$.

KON and GC occurred (Supplementary Figure S3), no fluorescence complementation was observed. Therefore, the origin of the fluorescent protein seems to be involved in the complementation ability of BiFC fragments.

\section{Acknowledgments}

We thank Atsushi Miyawaki (RIKEN, Japan), Satoshi Karasawa (MBL, Japan), and Takashi Fukano (RIKEN, Japan) for providing cDNA of $\mathrm{KO}$ variants and for valuable discussion. We also thank Minami Matsui (RIKEN, Japan) for giving us an opportunity to begin this work. This work was supported by the NOVARTIS Foundation (Japan) for the Promotion of Science (Y.K.), the JST-ERATO (JPMJER1602: the Numata Organelle Reaction Cluster) (Y.K.), the Japan Society for the Promotion of Science (JSPS) KAKENHI (No. 26840088) (Y.K.), and the CDI Research Project of Utsunomiya University (Y.K).

\section{Author contributions}

YK conceived and supervised the study; YF and AY performed experiments; YF and YK wrote the manuscript.

\section{Competing interests}

The authors declare no competing interests.

\section{References}

1. Hu CD, Chinenov Y, and Kerppola TK. Visualization of interactions among bZIP and Rel family proteins in living cells using bimolecular fluorescence complementation. Mol. Cell 9, 789-798 (2002).

2. Kodama Y, Wada M. Simultaneous visualization of two protein complexes in a single plant cell using multicolor fluorescence complementation analysis. Plant Mol. Biol. 70, 211-217 (2009).

3. Kodama Y, Hu CD. Bimolecular fluorescence complementation (BiFC): A 5-year update and future perspectives. BioTechniques 53, 285-298 (2012).

4. Filonov GS, Verkhusha VV. A near-infrared BiFC reporter for in vivo imaging of protein-protein interactions. Chem. Biol. 20, 1078-1086 (2013).

5. Karasawa S, Araki T, Nagai T, Mizuno H, Miyawaki A. Cyan-emitting and orange-emitting fluorescent proteins as a donor/acceptor pair for fluorescence resonance energy transfer. Biochem. J. 381, 307-312 (2004).

6. Sakaue-Sawano A, Kurokawa H, Morimura T et al. Visualizing spatiotemporal dynamics of multicellular cell-cycle progression. Cell 132, 487-498 (2008).

7. Chudakov DM, Matz MV, Lukyanov S, Lukyanov KA. Fluorescent proteins and their applications in imaging living cells and tissues. Physiol. Rev. 90, 1103-1163 (2010)

8. Fujii $Y$, Kodama Y. In planta comparative analysis of improved green fluorescent proteins with reference to fluorescence intensity and bimolecular fluorescence complementation ability. Plant Biotechnol. 32, 81-87 (2015).

9. Wu FH, Shen SC, Lee LY, Lee SH, Chan MT, Lin CS. Tape-Arabidopsis sandwich - a simpler Arabidopsis protoplast isolation method. Plant Methods 5, 16 (2009).

10. Kodama Y. A bright green-colored bimolecular fluorescence complementation assay in living plant cells. Plant Biotechnol. 28, 95-98 (2011).

11. Schneider CA, Rasband WS, Eliceiri KW. NIH Image to ImageJ: 25 years of image analysis. Nat. Methods 9, 671-675 (2012).

12. Kodama $\mathrm{Y}, \mathrm{Hu} \mathrm{CD}$. Bimolecular fluorescence complementation (BiFC) analysis of proteinprotein interaction: How to calculate signal-tonoise ratio. Methods Cell Biol. 113, 107-121 (2013). 
13. Ihaka R, Gentleman R. R: a language for data analysis and graphics. J. Comp. Graph. Stat. 5, 299-314.

14. Ohashi K, Kiuchi T, Shoji K, Sampei K, Mizuno K. Visualization of cofilin-actin and Ras-Raf interactions by bimolecular fluorescence complementation assays using a new pair of split Venus fragments. BioTechniques 52, 45-50 (2012).

15. Zhou J, Lin J, Zhou C, Deng X, Xia B 2011. An improved bimolecular fluorescence complementation tool based on superfolder green fluorescent protein. Acta Biochim. Biophys. Sin 43, 239-244.

16. Kodama $Y$, Shinya $T$, Sano $H$. Dimerization of $\mathrm{N}$-methyltransferases involved in caffeine biosynthesis. Biochimie 90, 547-551 (2008).

17. Hynes TR, Yost E, Mervine S, Berlot $\mathrm{CH}$. Multicolor BiFC analysis of competition among $\mathrm{G}$ protein beta and gamma subunit interactions. Methods 45, 207-213 (2008).

18. Vidi PA, Chemel BR, Hu CD, Watts VJ. Liganddependent oligomerization of dopamine $\mathrm{D}(2)$ and adenosine $\mathrm{A}(2 \mathrm{~A})$ receptors in living neuronal cells. Mol. Pharmacol. 74, 544-551 (2008).

19. Xing S, Wallmeroth N, Berendzen KW, Grefen C. Techniques for the analysis of protein-protein interactions in vivo. Plant Physiol. 171, 727-758 (2016).

20. Kodama Y. Time gating of chloroplast autofluorescence allows clearer fluorescence imaging in planta. PLoS ONE 11, e0152484 (2016).

21. Jach G, Pesch M, Richter K, Frings S, Uhrig JF. An improved mRFP1 adds red to bimolecular fluorescence complementation. Nat. Methods 3, 597-600 (2006).
First draft submitted: 3 July 2017; Accepted for publication: 31 January 2018

Address correspondence to Y. Kodama, Center for Bioscience Research and Education, Utsunomiya University, Tochigi 321-8505, Japan.

E-mail: kodama@cc.utsunomiya-u.ac.jp

To purchase reprints of this article, contact: s.cavana@future-science.com 